\title{
Preface: Special Issue on Recent Advances in Variational Calculus: Principles, Tools and Applications
}

\author{
Annamaria Barbagallo ${ }^{1} \cdot$ Lina Mallozzi $^{1} \cdot$ Michel Théra $^{2,3}$ \\ Published online: 23 May 2019 \\ (c) Springer Science+Business Media, LLC, part of Springer Nature 2019
}

This special issue is comprised of eighteen articles that investigate various aspects of variational calculus. Contributions focus on both the abstract and numerical point of view with major applications in optimization theory and methods.

Potentially, we may cite, for instance, the advancement of the general theory of regularity and stationarity that have recently emerged, like that of metric regularity or error bounds. These regularity concepts are appropriate for studying stability of solutions to optimization problems, particularly those of semi-infinite optimization and programs with equilibrium constraints, when standard assumptions are not satisfied.

We may also provide in a large part of this issue some contributions establishing new connections between the theoretical estimates for several regularity properties within the field of variational analysis and convergence analysis of computational algorithms, enhancing the existing applied models, computational algorithms and facilitating the post-optimal analysis of solutions of variational systems.

The range of applicability of this results is wide, for example, we mention applications in management (facility location, energy policy), engineering (optimal design, robotics), environmental sciences, as well as in many fields of economics (oligopolistic markets, network design, electric power pricing) and in emerging areas such as signal processing, finance, risk and statistical learning.

Annamaria Barbagallo

annamaria.barbagallo@unina.it

Lina Mallozzi

mallozzi@unina.it

Michel Théra

michel.thera@unilim.fr

1 Dipartimento di Matematica e Applicazioni, Università di Napoli Federico II, Naples, Italy

2 XLIM UMR-CNRS 7252, Université de Limoges, Limoges, France

3 Centre for Informatics and Applied Optimisation (CIAO), Federation University Australia, Ballarat, Australia 
The issue consists of the following eighteen articles:

1. New Farkas-type results for vector-valued functions: a non-abstract approach by N. Dinh, M. A. Goberna, D. H. Long, M. A. López, which provides new Farkastype results;

2. Asymptotic equivalence of evolution equations governed by cocoercive operators and their forward discretizations, by A. Contreras, J. Peypouquet, which studies discrete approximations of evolution equations governed by cocoercive operators by means of Euler iterations, both in a finite and in an infinite time horizon;

3. Nonsmooth and nonconvex optimization via approximate difference-of-con-vex decompositions, by W. van Ackooij, W. de Oliveira, which proposes an optimization technique for computing stationary points of a broad class of non-smooth nonconvex programming problems;

4. Metric and geometric relaxations of self-contracted curves, by A. Daniilidis, R. Deville, E. Durand-Cartagena, which extends the metric notion of a self-contracted curve;

5. The proximal alternating minimization algorithm for two-block separable convex optimization problems with linear constraints, by S. Bitterlich, R. I. Bot, E. Csetnek, which uses the alternating minimization algorithm to solve convex programming problems applied in image processing and machine learning;

6. Lipschitz modulus of the optimal value in linear programming, by M. J. Gisbert, M. J. Canovas, J. Parra, F. J. Toledo, devoted to the computation of the Lipschitz modulus of the optimal value function restricted to its domain in linear programming under different type of perturbations;

7. Second-order time and state dependent sweeping process in Hilbert space, by $\mathrm{F}$. Aliouane, D. Azzam-Laouir C. Castaing, D. M. Montero Marques, which studies second order perturbed Moreau's sweeping processes;

8. On characterizations of submanifolds via smoothness of the distance function in Hilbert spaces, by D. Salas, L. Thibault, which characterizes some classes of submanifolds of Hilbert spaces via some smoothness properties of square distance functions;

9. Extremality, stationarity and generalized separation of collections of sets, by $\mathrm{H}$. T. Bui, A. Kruger, that provides a unifying theoretical presentation of the extremal principle;

10. Surjectivity in Fréchet spaces, by M. Ivanov, N. Zlateva, which proves surjectivity results in Frèchet spaces of Nash-Moser type;

11. On generalized Bolza problem and its application to dynamic optimization, by A. D. Ioffe, that studies properties and mutual relationship of unconstrained variational problems of Bolza type and optimal control problems with state constraints for systems governed by differential inclusions;

12. Scalarization functionals with uniform level sets in set optimization, by T. Q. Bao, C. Tammer, which characterizes upper and lower set-less minimizers of some set valued mappings;

13. Directional Pareto efficiency: concepts and optimality conditions, by T. Chelmus, M. Durea, E. A. Florea, where a notion of directional Pareto minimality with respect to a set is introduced, generalizing the classical concept of Pareto efficiency; 
14. A further study on asymptotic functions via variational analysis, by F. Lara, R. López, B. F. Svaiter, which introduces and examines the upper and lower asymptotic operators characterizing its fixed points and zeros;

15. A new variational approach to linearisation of traction problems in elasticity, by F. Maddalena, D. Percivale, F. Tomarelli which studies a new energy functional for pure traction problems in elasticity;

16. Towards tractable constraint qualifications for parametric optimisation problems and applications to generalized Nash games, by D. Aussel, A. Svensson which studies generalized Nash games, where both the objective function and the feasible set depend on the variables of the other players;

17. Multiple capture of given number of evaders in linear recurrent differential games, by N. Petrov, N. Solov'eva which deals with the linear pursuit problem with $n$ pursuers and $m$ evaders with equal opportunities for all participants and geometric restrictions on the control of players;

18. Variational analysis down under open problem session, by H. T. Bui, S. B. Lindstrom, V. Roshchina, which presents some open questions in variational analysis.

We want to express our gratitude to Professor Franco Giannessi for having provided us with the opportunity to publish this special issue of JOTA, to the authors for having submitted articles of considerable interest and to the referees who reviewed the papers with competence and in a timely manner.

We hope that you will enjoy reading this issue containing new perspectives and opening new research directions.

The guest editors,

Annamaria Barbagallo, Napoli

Lina Mallozzi, Napoli

Miche Théra, Limoges

Publisher's Note Springer Nature remains neutral with regard to jurisdictional claims in published maps and institutional affiliations. 